\title{
Inducing micromechanical motion by optical excitation of a single quantum dot
}

\author{
Jan Kettler ${ }^{1,2}$, Nitika Vaish ${ }^{1,2}$, Laure Mercier de Lépinay ${ }^{1}$, Benjamin Besga ${ }^{3}$, Pierre-Louis \\ de Assis ${ }^{1,2,4}$, Olivier Bourgeois ${ }^{1,2}$, Alexia Auffèves ${ }^{1,2}$, Maxime Richard ${ }^{1,2}$, Julien Claudon ${ }^{5}$, \\ Jean-Michel Gérard ${ }^{5}$, Benjamin Pigeau ${ }^{1}$, Olivier Arcizet ${ }^{1},{\text { Pierre } \text { Verlot }^{6} \text {, and Jean-Philippe Poizat }}^{1,2}$ \\ 1 Univ. Grenoble Alpes, CNRS, Grenoble INP, Institut NEEL, France \\ ${ }^{2}$ CNRS, Inst. NEEL, "Nanophysique et semiconducteurs" group, Grenoble, France \\ 3 Univ Lyon, CNRS, Laboratoire de Physique, ENS de Lyon, France \\ 4 Gleb Wataghin Institute of Physics, University of Campinas, São Paulo, Brazil \\ 5 CEA, Univ. Grenoble Alpes, IRIG-PHELIQS, \\ "Nanophysique et semiconducteurs" group, France \\ 6 School of Physics and Astronomy, University of Nottingham, United Kingdom
}

\begin{abstract}
Hybrid quantumoptomechanical systems [1] interface a macroscopic mechanical degree of freedom with a single two-level system (TLS) such as a single spin [2-4], a superconducting qubit [5-7], or a single optical emitter [8-12]. Recently, hybrid systems operating in the microwave domain have witnessed impressive progress $[13,14]$. Concurrently, only a few experimental approaches successfully addressed hybrid systems in the optical domain, where macroscopic motion can modulate the TLS transition energy $[9,10,15]$. However, the reciprocal effect, corresponding to the backaction of a single quantum system on a macroscopic mechanical resonator, has remained elusive. In contrast to optical cavities, a TLS operates with no more than a single energy quantum. Hence, it requires a much stronger hybrid coupling rate compared to cavity optomechanical systems $[1,16]$. Here, we us a hybrid system made of a microwire whose motion couples to a single embedded semiconductor quantum dot (QD) via strain [9]. We resonantly drive the QD's exciton using a laser modulated at the mechanical frequency. In turn, the time-dependent excitation actuates the microwire motion. The mechanical force, which originates from state-dependent strain, is almost three orders of magnitude larger than the radiation pressure produced by the photon flux interacting with the QD. In principle, the state-dependent force could constitute a strategy to coherently encode the QD quantum state onto a mechanical degree of freedom [1].
\end{abstract}

The hybrid system we use in this work (see Fig. 1a) is composed of a single InAs quantum dot (QD) embedded close to the basis of a conical GaAs microwire $[9,19]$. The QD can be considered as a TLS with a ground state $|g\rangle$, and an excited state $|e\rangle$ corresponding to a trapped exciton. The energy of the TLS optical transition is coupled to the flexural vibration of the wire via strain-induced semiconductor band gap corrections [8, 9, 15, 20-24]. Inspired by a recent proposal based on this coupling [25], we employ here optical excitation to modulate the exciton population at the mechanical frequency: the resulting periodic strain induced by the QD then generates a flexural oscillation of the wire. This effect originates from the interaction between the individual exciton created in the $\mathrm{QD}$ and the crystalline lattice via the deformation potential $[8,25]$. The presence of an exciton in the QD generates an additionnal strain compared to the empty QD. This state-dependent strain produces in turn a flexion of the whole wire.

The dynamics of this hybrid strain-coupled system is described by the parametric Hamiltonian,

$H=\hbar \omega_{0} \frac{\left(\hat{\sigma}_{z}+1\right)}{2}+\hbar \Omega_{m}\left(\hat{b}^{\dagger} \hat{b}+1 / 2\right)+\hbar g_{m} \frac{\left(\hat{\sigma}_{z}+1\right)}{2}\left(\hat{b}+\hat{b}^{\dagger}\right)$,

where $\hbar \omega_{0}$ is the QD transition energy for the wire at rest $\left(\hbar \omega_{0} \simeq 1.35 \mathrm{eV}\right.$, wavelength $\left.920 \mathrm{~nm}\right)$, and $\hat{\sigma}_{z}=$ $|e\rangle\langle e|-| g\rangle\langle g|$ is the Pauli operator describing the population of the QD states. $\Omega_{m} / 2 \pi$ is the mechanical eigenfrequency of one of the wire fundamental flexural modes
$\left(\Omega_{m} / 2 \pi=330 \mathrm{kHz}\right.$, see Fig. $\left.1 \mathrm{~b}\right)$, and $\hat{b}$ the phonon annihilation operator of this mode. The last term of Eq.(1) describes the strain-mediated coupling, and can be rewritten as $\hbar g_{m} \frac{\left(\hat{\sigma}_{z}+1\right)}{2} \frac{\hat{x}}{x_{\mathrm{zpf}}}$. Here, $\hat{x}=x_{\mathrm{zpf}}\left(\hat{b}+\hat{b}^{\dagger}\right)$ is the top facet position of the wire, with $x_{\mathrm{zpf}}=\sqrt{\hbar / 2 m \Omega_{m}}$ the corresponding zero point fluctuations and $m$ the microwire effective mass associated with the flexural mode. The coupling strength reads $g_{m}=\frac{d \omega_{0}}{d x} x_{\text {zpf }}$. Following [9], we have measured $\frac{d \hbar \omega_{0}}{d x}=10 \mu \mathrm{eV} / \mathrm{nm}$ from the QD photoluminescence lineshape broadening when flexural motion is excited (see SI), while $m=32 \mathrm{pg}$ and $x_{\mathrm{zpf}}=28 \mathrm{fm}$ are deduced from Brownian motion measurements (see Fig. 1b and SI). For the chosen QD, this yields $g_{m} / 2 \pi=68 \mathrm{kHz}$.

The interaction between the microwire and the QD relies on the strain difference that the material surrounding the QD experiences when the QD hosts a single exciton [26]. The rest position $\mathrm{x}_{e}$ of the mechanical oscillator for the excited QD is different from the rest position $\mathrm{x}_{g}$ for the empty QD (see Fig. 1c,d and SI). The difference between these two positions is given by

$$
\mathrm{x}_{e}-\mathrm{x}_{g}=-2 \frac{g_{m}}{\Omega_{m}} x_{\mathrm{zpf}} .
$$

As a consequence, when the QD is in the ground state and is optically brought in the excited state on a time scale much faster that $2 \pi / \Omega_{m}$, the $\mathrm{QD}$ induces on the wire a static force

$$
F_{\mathrm{QD}}=k\left(\mathrm{x}_{e}-\mathrm{x}_{g}\right),
$$




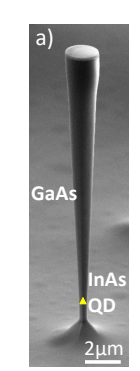

b)
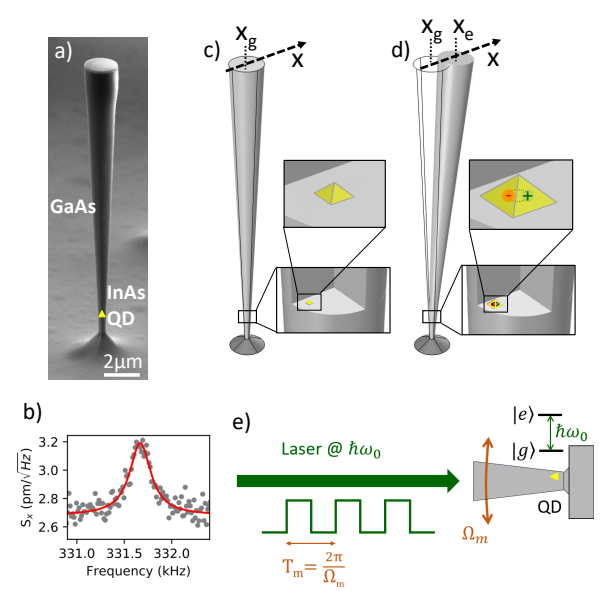

Figure 1: Principle. a, Scanning electron microscope image of the GaAs photonic wire. The approximate position of the InAs QD is marked by a yellow triangle. b, Brownian motion of the wire at cryostat temperature of $T=5 \mathrm{~K}$ (microwire temperature $T=25 \mathrm{~K}$, see SI) measured with a probe laser intensity $P=100 \mu \mathrm{W}$ as in the experiment. $\mathbf{c}, \mathbf{d}$, Schematic explanation of the effect. The lower insets are a cut of the wire showing the approximate position of the QD (yellow pyramid). When the QD is in its ground state (in c), the wire rest position is $\mathrm{x}_{g}$. When the QD is excited (hosts an exciton, represented as an orange electron and a green hole in d), its physical size is larger so that the rest position of the wire is displaced to $\mathrm{x}_{e}$. e, Principle of the experiment. The QD is illuminated by a laser resonant with its optical transition at $\hbar \omega_{0}$. The laser intensity is modulated at the wire mechanical frequency $\Omega_{m} / 2 \pi$, and the wire motion is measured as a function of the laser detuning with respect to the QD transition.

with $k=m \Omega_{m}^{2}$ the oscillator stiffness.

In our experiment, the QD is driven by a laser whose intensity is chopped with a $50 \%$ duty cycle at the wire's mechanical frequency $\Omega_{m} / 2 \pi$. The QD exciton decay time typically amounts to $\tau_{\mathrm{QD}}=1 \mathrm{~ns}$ [27], about 3 orders of magnitude faster than the mechanical oscillator period $(\sim 0.3 \mu \mathrm{s})$. Thus, the force generating the mechanical motion only depends on the average QD population measured by $\left\langle\hat{\sigma}_{z}\right\rangle$ over half a period. When the laser is on (off) the maximum average excited state population is $1 / 2(0)$, so that $\left\langle\hat{\sigma}_{z}\right\rangle=0\left(\left\langle\hat{\sigma}_{z}\right\rangle=-1\right)$. So the wire rest position is periodically displaced from $\mathrm{x}_{g}$ to $\mathrm{x}_{g}+\left(\mathrm{x}_{e}-\mathrm{x}_{g}\right) / 2$, leading to the excitation of a $\mathrm{QD}$ induced wire motion (Fig. 1e) with a root mean square (rms) amplitude at resonance (see SI):

$$
x_{\mathrm{QD}}=\frac{\sqrt{2}}{\pi} \eta \frac{g_{m}}{\Omega_{m}} Q x_{\mathrm{zpf}},
$$

where $\eta$ is an efficiency factor accounting for the fact that photons reaching the QD may not all excite it due e.g. to spectral wandering of the transition line, and $Q$ is the wire mechanical quality factor $(Q=1650)$. For $\eta=1$,

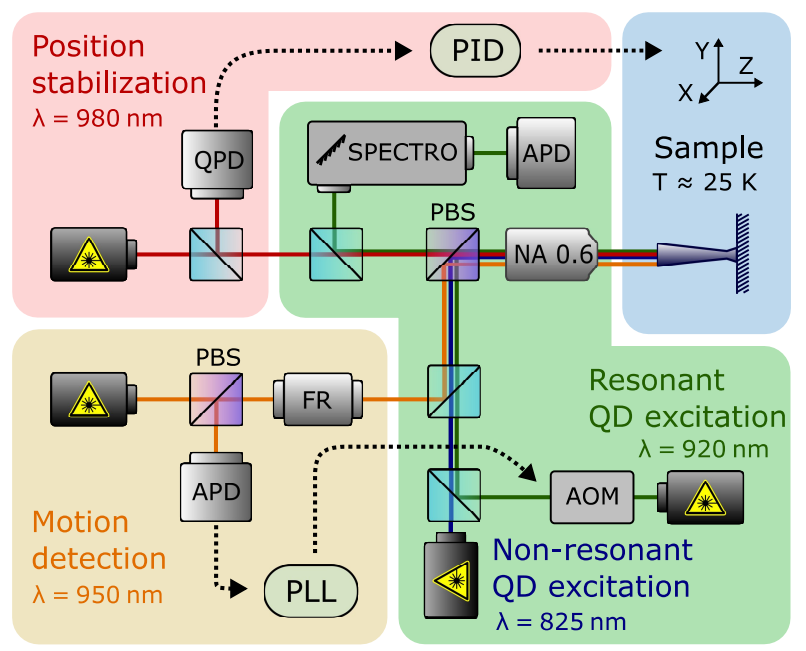

Figure 2: Experimental set-up. The sample is anchored on the cold finger of a helium flow cryostat. The sample temperature is $T=25 \mathrm{~K}$. The light enters the microscope objective of numerical aperture $\mathrm{NA}=0.6$, via a polarizing beam splitter (PBS). Resonant QD excitation (green background) is performed by an external cavity diode laser at $\lambda=920$ $\mathrm{nm}$, which is intensity modulated at the wire resonant mechanical frequency by an acousto optical modulator (AOM). To remain on resonance, the modulation frequency is locked on the photothermally actuated motion via a phase lock loop (PLL). A non resonant diode laser at $\lambda=825 \mathrm{~nm}$ is used for preliminary photoluminescence characterization, and as a "cleaning" laser for the resonant excitation (see Methods). Fluorescence photons are counted by an Avalanche Photodiode (APD) at the output of a high resolution $(12 \mu \mathrm{eV})$ spectrometer. Motion detection (orange background) is carried out with an external cavity diode laser at $\lambda=950 \mathrm{~nm}$. Its position dependent reflexion from the wire top facet is extracted using a Faraday rotator (FR) and sent to a photodiode whose signal is demodulated with a lock-in amplifier (see Methods). The position stabilization (pink background) is performed with a diode laser at $\lambda=980 \mathrm{~nm}$, whose reflexion from the wire top facet is detected on a quadrant photodiode (QPD) feeding a Proportional Integral Derivative (PID) servo controlling the microscope objective $\mathrm{x}-\mathrm{y}$ position. Lasers at $\lambda=920,950,980 \mathrm{~nm}$ are intensity stabilized.

our system parameters lead to $x_{\mathrm{QD}}^{\eta=1}=4.4 \mathrm{pm}$, whereas the rms spread of the Brownian motion at a temperature $T=25 \mathrm{~K}$ is $x_{B r}=x_{\mathrm{zpf}} \sqrt{2 k_{B} T / \hbar \Omega_{m}}=50 \mathrm{pm}$.

The experimental set-up (see Fig. 2) combines a resonant fluorescence set-up with a motion measurement scheme (see Methods). The excitation laser is intensity modulated by an acousto-optical modulator (AOM) at the mechanical frequency with an on-off square function. Its continuous wave intensity before the microscope ob- 
jective is $150 \mathrm{nW}$, slightly above the QD saturation power of $100 \mathrm{nW}$. A characteristic property of QD induced motion is that it features an amplitude proportional to the QD excited state population, which is monitored by the QD fluorescence. To reveal this signature, we scan the excitation laser frequency across the QD resonance while measuring simultaneously the QD fluorescence and the wire motion (see Methods). The latter is demodulated at the driving mechanical frequency to determine its two quadratures. The incoherent Brownian motion is averaged out by acquiring data for more than 20 hours, while laser intensities and microwire position are actively stabilized (see SI).

The QD fluorescence is plotted as a function of laser detuning in Fig. 3a, together with the wire motion amplitude in Fig. 3b. The averaged motion amplitude features a peak which perfectly matches the resonance condition between the laser and the QD transition. This constitutes a first signature of the QD induced motion. The motion peak lays on top of a 50 pm non-resonant background. Since the laser power is sufficiently low to avoid any significant radiation pressure or gradient force, we attribute this background to photothermal actuation of the wire [24] (see Methods).

A second signature of the QD induced motion is related to its phase. As seen in Fig. 3c,e the photothermal motion features a phase delay $\Phi_{P T}=-36^{\circ}$ between the excitation and the force. This delay is attributed to heat conduction and dissipation within the wire (see SI). On the other hand, the QD induced force is expected to be quasi-instantaneous as it follows the comparatively fast QD dynamics $\left(\tau_{\mathrm{QD}} \ll 2 \pi / \Omega_{m}\right)$. The total coherent motion resulting from the coherent addition of these two actuation mechanisms experiences therefore a phase shift $\Delta \Phi_{Q D}$ with respect to the photothermal motion alone (see Fig. 3d). This phase shift is indirectly measured via a phase locked loop (PLL) used to track the mechanical frequency (see Methods and SI for details). As seen in Fig. 3c the inferred averaged motion phase shift exhibits a peak at $\mathrm{QD}$ resonance, whose origin is the QD induced motion. The rms coherent motion vector extremities for detunings scanned across the QD resonance are displayed in Fig. 3e in the quadratures plane. The vertical orientation of these points indicates that the QD induced force follows instantaneously the laser modulation, in line with the fact that the QD induced motion is caused by a strain effect governed by the fast QD dynamics.

The experimental rms amplitude of the QD induced motion is $x_{\mathrm{QD}}=0.6 \pm 0.1 \mathrm{pm}$ as seen in Fig. 3e. This result matches the expected value, by adjusting $\eta$ to $\eta=0.15$ in Eq.(4). This non-ideal $\eta$ value is attributed to the fast (faster than $1 \mathrm{~s}$ ) spectral diffusion of the resonantly excited QD transition leading to an effective blinking with $15 \%$ of on periods as already observed with similar QDs [27].

The above measured displacement amplitude corresponds to a force $F_{\mathrm{QD}}^{\exp }=\frac{m \Omega_{m}^{2}}{Q} \times x_{\mathrm{QD}} \simeq 50 \mathrm{aN}$. We compare this force to the maximum radiation pressure force generated by a photon flux corresponding to the photon flux interacting with the QD in the present experiment. We therefore consider a laser beam with incident photon flux $\eta / 2 \tau_{\mathrm{QD}}$ perpendicular to the wire axis, and perfectly reflected at the very tip of the wire. This force is given by $F_{\text {rad }}=\eta \frac{\hbar k_{l}}{\tau_{\mathrm{QD}}}=0.1 \mathrm{aN}$, with $k_{l}$ the laser wave-vector, and $\tau_{\mathrm{QD}}=1 \mathrm{~ns}$ the $\mathrm{QD}$ lifetime.

Remarkably, this strong single-photon force enhancement compared to radiation force is granted while the underlying photon-exciton conversion process is preserving the photon number. This essential property makes our approach intrinsically suitable to quantum photonics engineering, including coherent processing of nonclassical quantum superpositions of QD states, and to the use of mechanics as a quantum bus for storing and routing entangled QD states. Such compelling applications require important technological improvements. Simulations show that optimizing and miniaturizing the geometry can increase both mechanical resonance frequency and hybrid coupling rate [30]. This strategy yields a triple benefit: i) At higher frequencies, the photothermal effect becomes much slower than the mechanical period and therefore vanishes. ii) Concurrently, this allows the system to operate in the resolved sideband regime $[31,32]$, which enables the preparation and manipulation of quantum motion states [8]. iii) Furthermore, higher coupling rates open the perspective to reach the strong coupling regime, were the coupling rate exceeds all decoherence rates.

Note that our result can be readily extended to existing systems, starting with waveguide embedded single photon sources [33], which may host ultra-high frequency mechanical degrees of freedom [34, 35]. Other potential high-frequency candidates include hybrid phononic nanostrings [36], surface acoustic waves coupled to quantum dots or nitrogen vacancy centers [20, 21], and hybrid photonic crystal cavities [37].

These improvements notwithstanding, our experiment can already be interpreted and exploited from the more fundamental standpoint of quantum thermodynamics, the whole process being reminiscent of a nanoengine [38]. The quantum emitter converts the energy incoherently provided by the light field (heat) into coherent mechanical motion (work). Importantly, the bath is outof-equilibrium, which opens novel scenarios for quantum and information thermodynamics [39, 40].

To summarize, we have been able to set in motion a micro-oscillator of mass $0.1 \mathrm{ng}$ by optically manipulating the quantum state of a single quantum object. The motion is generated by periodically driving the excitonic population of an embedded quantum dot, which is coupled via strain to the position of the oscillating wire. This built-in coupling is extremely robust to any external perturbation and features a high level of integration, making the next generation of hybrid photonic nanowires a potential solid-state building block for quantum photonics circuitry. 

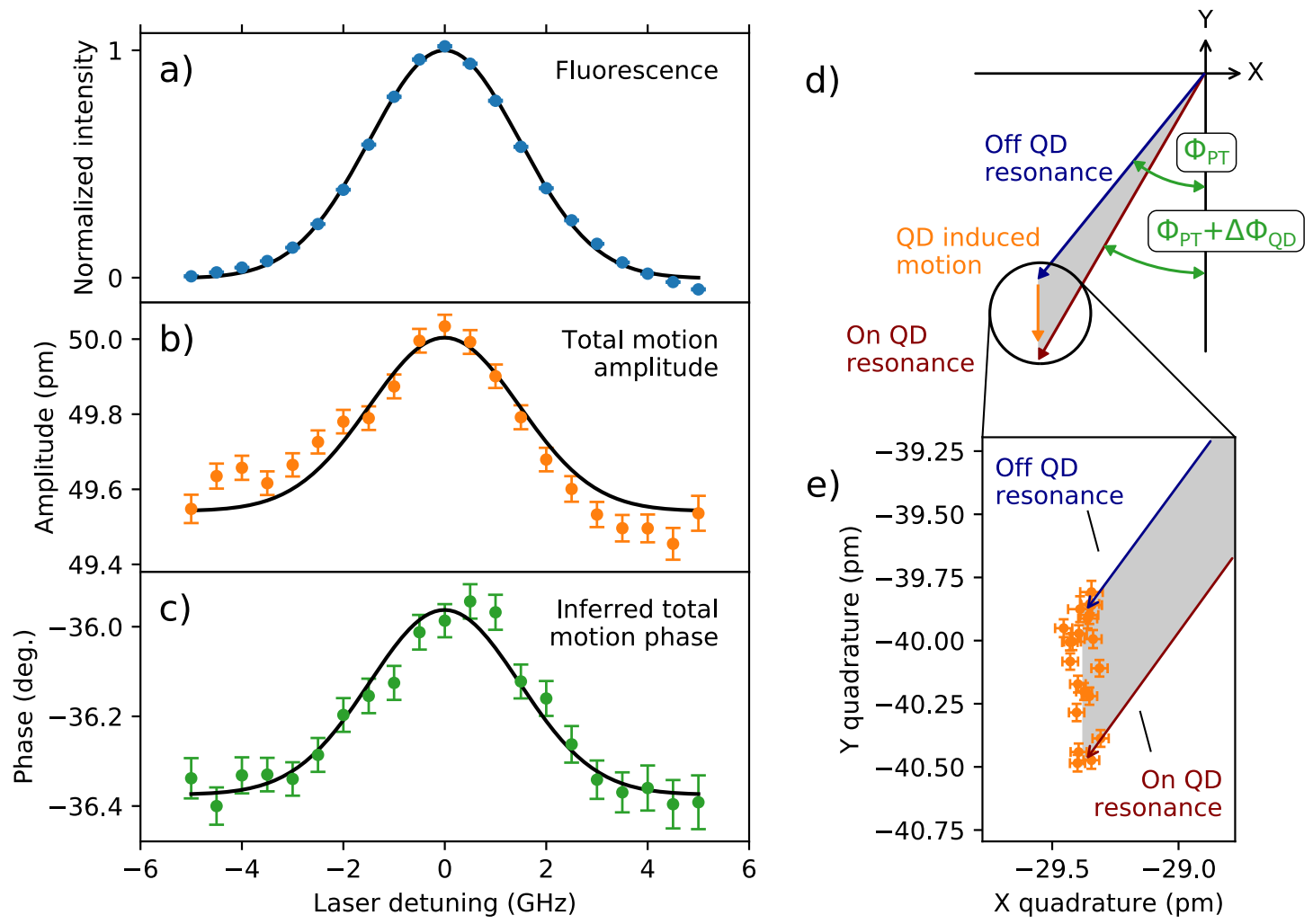

Figure 3: Experimental results. a, QD fluorescence, b, total motion rms amplitude, and c, total motion phase shift, are displayed as a function of the resonant laser detuning with respect to the QD transition. The fluorescence data is fitted with a Gaussian whose centering and width are kept for the fitting of the motion amplitude and the phase shift. In c, the phase shift is inferred from the frequency shift the PLL has to apply to maintain the set phase (see Methods and SI). d, This sketch represents the coherent component of the wire motion in the quadratures plane (rotating frame). $X$ and $Y$ are the rms values of the coherent motion quadratures, defined as $x_{c}(t)=\sqrt{2}\left(X \cos \Omega_{m} t-\sin \Omega_{m} t\right)$. The phase reference is the laser modulation $I_{\text {las }}(t)=I_{0} \cos \Omega_{m} t$. A phase delay is thus described with a negative angle. In blue is shown the total motion vector when the laser is off QD resonance. This corresponds to the photothermal (PT) motion only. The orange vector represents the QD induced motion at QD resonance. The red vector displays the total motion at QD resonance. It is recalled that for a drive with instantaneous action, the motion at the mechanical resonance exhibits a $-90^{\circ}$ phase shift. The PT motion features an extra $\Phi_{P T}=-36^{\circ}$ phase shift. In e are displayed experimental data corresponding to a zoom on the effect of the QD induced motion on the total motion. The photothermal background motion is represented by the blue vector. Each data point represents the total motion (ie photothermal + QD induced) corresponding to a different laser detuning across the QD resonance as in a-c. For all the graphs of this figure, error bars are the standard error of the mean.

\section{Acknowledgements}

JK, NV, AA, JC, JMG, PV and JPP are supported by the French National Research Agency (project QDOT ANR-16-CE09-0010). NV is supported by Fondation Nanosciences. PLdA thanks Université Grenoble Alpes and CNRS for supporting visits as invited scientist. AA is supported by the Agence Nationale de la Recherche under the Research Collaborative Project Qu-DICE (ANRPRC-CES47). MR is supported by the Agence Nationale de la Recherche under the Research Collaborative Project QFL (ANR-16-CE30-0021). BP is supported by the
Agence Nationale de la Recherche under the project QCForce (ANR-JCJC-2016-CE09). OA acknowledges support from ERC Atto-Zepto. PV acknowledges support from the ERC StG 758794 "Q-ROOT". Sample fabrication was carried out in the "Plateforme Technologique Amont" and in CEA/LETI/DOPT clean rooms.

\section{Author contributions}

JK and NV performed the experiments with the help of BB and PLA. JK and LML wrote the experimental 
codes. JK performed the data analysis. OB did the photothermal analysis of the system. AA and MR provided theoretical support. JC and JMG designed and fabricated the samples. JK, BP, OA, PV and JPP proposed the experimental procedures. JPP supervised the project and wrote the manuscript with the help of AA, MR, JC, JMG, BP, OA and PV.

\section{Competing interests}

The authors declare no competing interests.

\section{Methods}

\section{QD fluorescence detection}

The QD fluorescence (wavelength around $920 \mathrm{~nm}$ ) is detected by a photon counting avalanche photodiode at the output of a $1.5 \mathrm{~m}$ focal length spectrometer equipped with a 1200 grooves $/ \mathrm{mm}$ grating (resolution $12 \mu \mathrm{eV}$ ). The resonant laser reflexion is attenuated by a cross polarizer scheme. To further reject the resonant laser light, the spectrometer is adjusted on the high energy phonon side band $0.5 \mathrm{meV}$ away from the QD line [26]. Note that these high energy phonons $(0.5 \mathrm{meV}=120 \mathrm{GHz})$ are not at all related to the wire motion but to bulk semiconductor crystal thermal excitations. The phonon side band signal is lower than the zero phonon line, but the laser background is almost completely removed leading to a greatly improved signal to noise ratio (see SI).

Additionally, as already observed by other groups [41], the sample must be illuminated by a weak power (100 $\mathrm{nW}$ ) non resonant (wavelength $825 \mathrm{~nm}$ ) laser to saturate defects around the QD to reduce spectral diffusion and observe resonance fluorescence in good conditions.

Integrated over several hours, the QD linewidth is in the $100 \mu \mathrm{eV}$ range owing to slow spectral wandering on a time scale of several minutes. After proper data processing (see SI), we can reduce the effects of slow spectral wandering to reach a linewidth of $12 \mu \mathrm{eV}$ (see Fig. 3a).

Note that polarization sensitive photoluminescence spectroscopy of the chosen QD has shown that the line of interest comes from a charged exciton (trion).

\section{Wire motion detection}

The wire motion is detected via the reflexion of a laser focused on the edge of the wire top facet, so that the wire motion modulates the reflected intensity. This probe laser is a shot-noise limited external cavity diode laser. Its wavelength $(950 \mathrm{mn})$ is chosen so that it can be efficiently filtered from the QD fluorescence light using tunable edgepass interference filters, and its energy is below the QD transition energy to minimize its impact on QD excitation. The mechanical signal to noise ratio (in power) scales linearly with the probe laser intensity. However the detection of the QD resonance fluorescence is degraded for probe beam intensities exceeding $100 \mu \mathrm{W}$. We are thus limited to this intensity of $100 \mu \mathrm{W}$. This low light level requires the use of a high gain avalanche photodiode (APD) that enables us to measure the Brownian motion at a cryostat temperature of $T=5 \mathrm{~K}$ (sample temperature of $T=25 \mathrm{~K}$, see SI) in good conditions (see fig. 1b). Finally, for the displacement measurement to be quantitative, we carefully calibrate the APD voltage change $d V$ produced by a known static displacement $d x$ of the probe laser with respect to the wire (see SI).

\section{Photothermal motion}

The resonant laser is at an energy of $E_{l}=1.35 \mathrm{eV}$ (wavelength $920 \mathrm{~nm}$ ) well below the GaAs gap at $T=25$ $\mathrm{K}\left(E_{g}=1.518 \mathrm{eV}\right.$, wavelength: $\left.\lambda_{g}=817 \mathrm{~nm}\right)$. It is nevertheless very weakly absorbed along the wire, mainly by surface defects. Its on-off modulation at mechanical frequency leads to periodic heating and thus deformation of the non-perfectly symmetrical wire. From the QD line shift when the laser is "on", the temperature increase caused by light absorption has been estimated to be less than $0.01 \mathrm{~K}$. This QD independent driven motion has a root mean square amplitude of $x_{P T}=50 \mathrm{pm}$ and features a phase delay $\Phi_{P T}=-36 \pm 5^{\circ}$ with respect to a motion that would respond instantaneously to the excitation laser modulation. This phase delay is well accounted for by estimating the thermal time response of the wire (see SI).

Thanks to this photothermal actuation we can lock the laser modulation frequency to the wire mechanical resonance using a Phase Locked Loop (PLL) to cope with the slow mechanical frequency drift attributed to wire icing. Additionally, the PLL in-loop frequency signal is used to infer the total motion phase shift (see below and SI).

\section{Experimental procedure}

The bottom of the wire contains a layer of about 100 self-assembled QDs [42] (cf Fig. 1a and SI). Different QDs exhibit different transition energies, so that resonant excitation allows us to address a single QD. The asymmetrical section of the wire gives rise to two non-degenerate fundamental flexural mechanical modes. The chosen QD is off-centered (about one fifth of the radius) so that it experiences strain as the wire oscillates along one of the two linearly polarized fundamental flexural modes [43], but not too far out to remain well coupled to the guided optical mode of the wire, and immune to surface effects.

The experimental procedure consists in scanning the intensity modulated resonant laser wavelength across the QD transition, while recording the motion of the wire. The QD induced motion being smaller than the Brown- 
ian motion $\left(x_{\mathrm{QD}} / x_{B r} \sim 1 / 100\right)$, the signal must be integrated over time to average out the Brownian motion (see SI). The chosen strategy is to carry out more than 1000 scans of one minute over a duration of about 20 hours. The scan data are postprocessed before the final averaging allowing to reduce the effects of technical noises and QD instabilities (see SI).

To ensure stability of the experiment during this time, the power of all lasers is stabilized, the thermal drift of the wire position with respect to all lasers is tracked using an extra laser (wavelength $980 \mathrm{~nm}$ ) reflected on the wire towards a quadrant photodiode (see SI), and the mechanical frequency is tracked with a phase locked loop (PLL), (see SI).

\section{Phase shift measurement}

During the experiment the mechanical drive frequency is locked to the wire resonance using a Phase Locked Loop (PLL). The total motion phase change $\Delta \Phi_{Q D}$ caused by the QD induced motion is compensated by the PLL via the shift of its driving frequency by $\Delta \Omega / 2 \pi$ in order to re- main on the set phase. For frequency change smaller than the mechanical linewidth (i.e. $\Delta \Omega \ll \Omega_{m} / 2 Q$ ) the phase to frequency conversion factor at mechanical resonance is $d \Phi / d \Omega=2 Q / \Omega_{m}$, so that $\Delta \Phi_{Q D}=\left(2 Q / \Omega_{m}\right) \Delta \Omega$. This allows us to infer the QD induced motion phase change $\Delta \Phi_{Q D}$ from the measured frequency change $\Delta \Omega / 2 \pi$ (see SI for more details), as plotted as the green dots in Fig. 3c.

\section{Data availability}

Data are available from the public repository https: //zenodo.org/record/4118790\#.X5H6jZrgqV4.

\section{Code availability}

Data processing code is available from the public repository https://zenodo.org/record/4118790\# .X5H6jZrgqV4.
[1] Treutlein, P., Genes, C., Hammerer, K., Poggio, M., Rabl, P. Hybrid Mechanical Systems. Cavity Optomechanics. Quantum Science and Technology. M. Aspelmeyer, T. Kippenberg, F. Marquardt, Editors, (Springer), Berlin, Heidelberg (2014).

[2] Rugar, D., Budakian, R., Mamin, H.J. \& Chui, B.W. Single spin detection by magnetic resonance force microscopy. Nature 430, 329-332 (2004).

[3] Rabl, P. et al. Strong magnetic coupling between an electronic spin qubit and amechanical oscillator. Phys. Rev. $B$ 79, 041302 (2009).

[4] Arcizet, O. et al A single nitrogen-vacancy defect coupled to a nanomechanical oscillator. Nature Phys. 7, 879-883 (2011).

[5] LaHaye, M.D., Suh, J., Echternach, P.M., Schwab, K., \& Roukes, M.L. Nanomechanical measurements of a superconducting qubit. Nature 459, 960-964 (2009).

[6] O'Connell, A.D. et al. Quantum ground state and singlephonon control of a mechanical resonator. Nature 464, 697-703 (2010).

[7] Pirkkalainen, J.M. et al. Hybrid circuit cavity quantum electrodynamics with a micromechanical resonator. $\mathrm{Na}$ ture 494, 211-215 (2013).

[8] Wilson-Rae, I., Zoller, P., \& Imamoğlu, A. Laser cooling of a nanomechanical resonator mode to its quantum ground state. Phys. Rev. Lett. 92, 075507 (2004).

[9] Yeo, I. et al. Strain-mediated coupling in a quantum dotmechanical oscillator hybrid system. Nature Nanotech. 9, 106-110 (2014).

[10] Tian, Y., Navarro, P., \& Orrit, M. Single molecule as a local acoustic detector for mechanical oscillators. Phys. Rev. Lett. 113, 135505 (2014).

[11] Teissier, J., Barfuss, A., Appel, P., Neu, E., Maletinsky, $\mathrm{P}$. Strain coupling of a nitrogen-vacancy center spin to a diamond mechanical oscillator. Phys. Rev. Lett. 113, 020503 (2014).

[12] Lee, D.H., Lee, K.W., Cady, J.V., Ovartchaiyapong P., \& Bleszynski Jayich, A.C. Topical review: spins and mechanics in diamond J. Optics 19, 033001 (2017).

[13] Satzinger, J. et al. Quantum control of surface acousticwave phonons. Nature 563, 661-665 (2018).

[14] Chu, Y. et al. Creation and control of multi-phonon Fock states in a bulk acoustic-wave resonator. Nature $\mathbf{5 6 3}$, 666-670 (2018).

[15] Montinaro, M. et al. Quantum Dot Opto-Mechanics in a Fully Self-Assembled Nanowire. Nano Lett. 14, 4454 (2014).

[16] Aspelmeyer, M., Kippenberg, T.J., Marquardt, F. Cavity optomechanics. Rev. Mod. Phys. 86, 1391 (2014).

[17] Chan, J. et al. Laser cooling of a nanomechanical oscillator into its quantum ground state. Nature 478, 89-92 (2011).

[18] Riedinger, R. et al. Remote quantum entanglement between two micromechanical oscillators. Nature 556, 473477 (2018).

[19] Munsch, M. et al. Dielectric GaAs Antenna Ensuring an Efficient Broadband Coupling between an InAs Quantum Dot and a Gaussian Optical Beam. Phys. Rev. Lett. 110, $177402(2013)$

[20] Metcalfe, M., Carr, S.M., Muller, A., Solomon, G.S. and Lawall, J. Resolved Sideband Emission of InAs/GaAs Quantum Dots Strained by Surface Acoustic Waves. Phys. Rev. Lett. 105, 037401 (2010).

[21] Schülein, F.J.R. et al. Fourier synthesis of radiofrequency nanomechanical pulses with different shapes. Nature Nanotech. 10, 512-516 (2015).

[22] Golter, D.A. et al. Coupling a Surface Acoustic Wave to an Electron Spin in Diamond via a Dark State. Phys. 
Rev. X 6, 041060 (2016).

[23] Carter, S.G. et al. Spin-Mechanical Coupling of an InAs Quantum Dot Embedded in a Mechanical Resonator Phys. Rev. Lett. 121, 246801 (2018).

[24] Carter, S.G. et al. Tunable Coupling of a Double Quantum Dot Spin System to a Mechanical Resonator. Nano Lett. 19, 6166-6172 (2019).

[25] Auffèves A. \& Richard, M. Optical driving of macroscopic mechanical motion by a single two-level system Phys. Rev. A 90, 023818 (2014).

[26] Besombes, L., Kheng, K., Marsal, L., \& Mariette, H. Acoustic phonon broadening mechanism in single quantum dot emission. Phys. Rev. B 63, 155307 (2001).

[27] Munsch, M. et al. Resonant driving of a single photon emitter embedded in a mechanical oscillator. Nat. Comm. 8, 76 (2017).

[28] Höhberger Metzger, C. \& Karrai, K. Cavity cooling of a microlever. Nature 432, 1002-1005 (2004).

[29] von Lindenfels, D. et al. Spin Heat Engine Coupled to a Harmonic-Oscillator Flywheel. Phys. Rev. Lett. 123, 080602 (2019).

[30] Artioli, A. et al, Design of Quantum Dot-Nanowire Single-Photon Sources that are Immune to Thermomechanical Decoherence Phys. Rev. Lett. 123, 247403 (2019).

[31] Garcia-Sanchez, D. et al. Acoustic confinement in superlattice cavities. Phys. Rev. A 94, 033813 (2016).

[32] Eichenfield, M., Chan, J., Camacho, R.M., Vahala, K.J., \& Painter, O. Optomechanical crystals. Nature 462, 7882 (2009).

[33] Lodahl, P., Mahmoodian, S., \& Stobbe, S. Interfacing single photons and single quantum dots with photonic nanostructures. Rev. Mod. Phys. 87, 347 (2015).

[34] Gavartin, E. et al. Optomechanical Coupling in a TwoDimensional Photonic Crystal Defect Cavity. Phys. Rev. Lett. 106, 203902 (2011)

[35] Esmann, M. et al. Brillouin scattering in hybrid optophononic Bragg micropillar resonators at $300 \mathrm{GHz}$, Optica 6, 854-859 (2019).

[36] Vogele, A. et al. Quantum Dot Optomechanics in Suspended Nanophononic Strings Adv. Quantum Tech. 3, $1900102(2020)$

[37] Sun, S., Kim, H., Solomon, G.S. \& Waks. E. Strain tuning of a quantum dot strongly coupled to a photonic crystal cavity Appl. Phys. Lett. 103, 151102 (2013).

[38] Elouard, C., Richard, M. \& Auffèves, A. Reversible work extraction in a hybrid opto-mechanical system. New J. Phys. 17, 055018 (2015).

[39] Rossnagel, J. et al. A single-atom heat engine Science 352, 325-329 (2016).

[40] Klaers, J. Landauer's Erasure Principle in a Squeezed Thermal Memory. Phys. Rev. Lett. 122, 040602 (2019).

[41] Majumdar, A., Kim, E.D., \& Vǔckovíc, J. Effect of photogenerated carriers on the spectral diffusion of a quantum dot coupled to a photonic crystal cavity. Phys. Rev. $B$ 84, 195304 (2011).

[42] Marzin, J.-Y., Gérard, J.-M., Izraël, A., and Barrier, D. Photoluminescence of single InAs quantum dots obtained by self-organized growth on GaAs, Phys. Rev. Lett. 73, 716 (1994).

[43] de Assis, P.L. et al. Strain-gradient position mapping of semiconductor quantum dots. Phys. Rev. Lett. 118, 117401 (2017). 